\title{
A NOTE ON RIESZ POTENTIALS AND THE FIRST EIGENVALUE
}

\author{
JIE-CHENG CHEN
}

(Communicated by J. Marshall Ash)

\begin{abstract}
In this paper, we consider the boundedness of Riesz potentials on positively curved manifolds. As an application, we get the greatest lower bound of the essential spectrum of a positively curved manifold.
\end{abstract}

For a complete Riemannian manifold $M$ of dimension $n$, the first eigenvalue is defined by the variational formula

$$
\mu_{0}=\inf _{f} \frac{\int_{M}|\nabla f|^{2} d x}{\int_{M} f^{2} d x}
$$

where $f$ ranges over the smooth functions of compact support, and where $\nabla$ is the gradient of the Riemannian metric of $M$. If $\Delta$ denotes the negative of the Laplace-Beltrami operator of $M$ (and hence is a positive operator) then it is easy to show by spectral theory that $\mu_{0}$ is the greatest lower bound of the spectrum of $M$.

As known, if $V(M)$, the volume of $M$, is finite then $\mu_{0}=0$; if $V(M)$ is infinite then $\mu_{0}$ is generally different from 0 for negatively curved manifolds (see [2-5]) - this fact is very important in analysis. In this paper, we consider the boundedness of Riesz potentials on $M$; as an application, we get $\mu_{0}=0$ for positively curved manifolds.

For the Riesz potential $\Delta^{-\alpha / 2}$, we know that for $M=R^{n}$, the HardyLittlewood-Sobolev embedding theorem holds, i.e., $\left\|\Delta^{-\alpha / 2}(f)\right\|_{q} \leq C_{n, p, q}\|f\|_{p}$ $(1 / q=1 / p-\alpha / n, 0<\alpha<n, 1<p, q<\infty)$, see [7]; for a Cartan-Hadamard manifold $M$, the Strichartz-Lohoue theorem holds, i.e., $\left\|\Delta^{-\alpha / 2}(f)\right\|_{q} \leq$ $C_{n, p}\|f\|_{p} \quad(1<p<\infty, 0<\alpha<n)$, see [6,8]. But for positively curved manifolds, we have

Theorem. If $\operatorname{Ric}(M) \geq 0$ and $M$ is noncompact, then $\Delta^{-\alpha / 2}$ is $L_{p} \rightarrow L_{q}$ bounded iff $V_{x}(r) \geq C_{n} r^{n}$ and $1 / q=1 / p-\alpha / n$, where $1<p, q<\infty, 0<$ $\alpha<n$.

Here $V_{x}(r)$ is the volume of the geodesic ball with center $x$ and radius $r$. The theorem shows that for noncompact positively curved manifolds, $\Delta^{-\alpha / 2}$ is not bounded in general because $V_{x}(r) \geq C_{n} r^{n}$ is usually not true, for example, for $M=R^{1} \times S^{n-1}$.

Received by the editors April 30, 1990 and, in revised form, June 18, 1991.

1991 Mathematics Subject Classification. Primary 42C99; Secondary 58C40.

Key words and phrases. Riesz potential, the first eigenvalue, positively curved manifold. 
Proof of Theorem. We have (see [1, Chapter 1] or [8])

$$
\begin{gathered}
\Delta^{-\alpha / 2}(f)(x)=\int_{M} I_{\alpha}(x, y) f(y) d y, \\
I_{\alpha}(x, y)=\Gamma^{-1}\left(\frac{1}{2}\right) \int_{0}^{\infty} s^{\alpha / 2} h_{s}(x, y) s^{-1} d s,
\end{gathered}
$$

where $h_{s}(x, y)$ is the heat kernel. We know that (see [9])

$$
\begin{aligned}
& h_{s}(x, y) \leq C_{1} V_{x}^{-1}(\sqrt{s}) \exp \left(-c_{1} d^{2}(x, y) / s\right), \\
& h_{s}(x, y) \geq C_{2} V_{x}^{-1}(\sqrt{s}) \exp \left(-c_{2} d^{2}(x, y) / s\right),
\end{aligned}
$$

where $d(x, y)$ is the geodesic distance between $x$ and $y$, and

$$
V_{x}^{-1}(R) \geq C_{n}(R / r) V_{x}^{-1}(r), \quad V_{x}(r) \geq(r / R)^{n} V_{x}(R),
$$

where $R>r>0$. So

$$
\begin{aligned}
I_{\alpha}(x, y) & \geq C_{n, \alpha} \int_{0}^{\infty} s^{\alpha / 2} V_{x}^{-1}(\sqrt{s}) \exp \left(\frac{-c_{2} d^{2}(x, y)}{s}\right) \frac{d s}{s} \\
& \geq C_{n, \alpha} \int_{0}^{d^{2}(x, y)} s^{\alpha / 2} V_{x}^{-1}(\sqrt{s}) \exp \left(\frac{-c_{2} d^{2}(x, y)}{s}\right) \frac{d s}{s} \\
& =C_{n, \alpha} V_{x}^{-1}(d(x, y)) d^{\alpha}(x, y)
\end{aligned}
$$

and

$$
\begin{aligned}
I_{\alpha}(x, y) \leq & C_{n, \alpha} \int_{0}^{\infty} s^{\alpha / 2} V_{x}^{-1}(\sqrt{s}) \exp \left(\frac{-c_{1} d^{2}(x, y)}{s}\right) \frac{d s}{s} \\
\leq & C_{n, \alpha}\left(\int_{0}^{d^{2}(x, y)} s^{\alpha / 2} V_{x}^{-1}(\sqrt{s})\left(\frac{d(x, y)}{\sqrt{s}}\right)^{n} \exp \left(\frac{-c_{1} d^{2}(x, y)}{s}\right) \frac{d s}{s}\right. \\
& \left.\quad+\int_{d^{2}(x, y)}^{\infty} s^{\alpha / 2} V_{x}^{-1}(\sqrt{s})\left(\frac{d(x, y)}{\sqrt{s}}\right) \exp \left(\frac{-c_{1} d^{2}(x, y)}{s}\right) \frac{d s}{s}\right) \\
\leq & C_{n, \alpha} V_{x}^{-1}(d(x, y)) d^{\alpha}(x, y)
\end{aligned}
$$

for $0<\alpha<1$. Now, if $V_{x}(r) \geq C_{n} r^{n}$ and $1 / q=1 / p-\alpha / n$, we can easily prove

$$
\left\|\Delta^{-\alpha / 2}(f)\right\|_{q} \leq C_{n, p}\|f\|_{p}
$$

for $0<\alpha<1$ by an easy modification of the proof of Theorem 1 of Chapter $\mathrm{V}$ of [7]. Then an iterative process yields the general result for $0<\alpha<n$. Conversely, if $\Delta^{-\alpha / 2}$ is $L_{p} \rightarrow L_{q}$ bounded, taking $f=\chi_{B\left(x_{0}, r\right)}$ where $x_{0}$ is a fixed point of $M$, we can get

$$
V_{x_{0}}(r)^{1 / p} \geq C_{n, p, q} r^{\alpha} V_{x_{0}}(r)^{q}
$$

because for $x \in B\left(x_{0}^{*}, r\right)$ where $x_{0}^{*}$ is a suitable point such that $d\left(x_{0}, x_{0}^{*}\right)=$ $3 r$; hence we have $\Delta^{-\alpha / 2}(f)(x) \geq C_{n, \alpha} r^{\alpha}$ and $V_{x_{0}}(r) / C_{n} \leq V_{x_{0}}(r) \leq C_{n} V_{x_{0}}(r)$. So

$$
V_{x_{0}}^{1 / p-1 / q}(r) \geq C_{n, p, q} r^{\alpha} \text {. }
$$

Now $V_{x_{0}}(r) \sim r^{n}(r \rightarrow 0)$ and $V_{x_{0}}(r) \lesssim r^{n}(r \rightarrow \infty)$, thus $1 / p-1 / q$ must be $\alpha / n$ and $V_{x_{0}}(r) \geq C_{n, p, q} r^{n}$. 
Corollary. If $\operatorname{Ric}(M) \geq 0$ then $\mu_{0}=0$.

Proof. It is known for $V(M)<\infty$. Now suppose $V(M)=+\infty$, i.e., $M$ is noncompact. If $\mu_{0}>0$, we have $\mu_{0}^{-1}\|\nabla(f)\|_{2} \geq\|f\|_{2}$. Taking $f=\Delta^{-1 / 2}(g)$, we get

$$
\left\|\Delta^{-1 / 2}(g)\right\|_{2} \leq C_{n}\left\|\nabla\left(\Delta^{-1 / 2}\right)(g)\right\|_{2} \leq C_{n}\|g\|_{2}
$$

by the $L^{2}$-boundedness of the Riesz transform (see [8]). This means that the Riesz potential $\Delta^{-1 / 2}$ is $L^{2}$-bounded in contraction to the above theorem.

Since $\mu_{0}=0$ is not an eigenvalue, the above corollary also shows that $\mu_{0}=0$ is just the greatest lower bound of the essential spectrum of $M$.

\section{ACKNOWLEDGMENT}

I would like to express my many thanks to the referee for his helpful and careful comments and corrections.

\section{REFERENCES}

1. J.-Ch. Chen, Heat kernels on positively curved manifolds, Ph.D. Thesis, Hangzhou Univ., 1987.

2. J.-Ch. Chen and J.-Y. Li, A note on eigenvalues, Chinese Sci. Bull. 34 (1989); or J.-Ch. Chen, On a conjecture of $S . T$. Yau, ibid. 33 (1988). (Chinese)

3. H. Donnelly, On the essential spectrum of a complete Riemannian manifold, Topology 20 (1981), 1-24.

4. J. F. Escobar, On the spectrum of the Laplacian on complete Riemannian manifolds, Comm. Partial Differential Equations 11 (1986), 63-68.

5. H. P. Mckean, An upper bound to the spectrum of $\Delta$ on a manifold of negative curvature, J. Differential Geom. 4 (1970), 359-366.

6. N. Lohoue, Puissances complexes de l'operateur de Laplace Beltrami, C. R. Acad. Sci. Paris Ser. A 290 (1980), 605-608.

7. E. M. Stein, Singular integrals and differentiability properties of functions, Princeton Univ. Press, Princeton, NJ, 1970.

8. R. S. Strichartz, Analysis of the Laplacian on the complete Riemannian manifold, J. Funct. Anal. 52 (1983), 48-79.

9. R. Schoen and S. T. Yau, Differential geometry, Chinese Academic Press, 1988. (Chinese)

Department of Mathematics, Hangzhou University, Hangzhou 310028, People's REPUBLIC OF CHINA 